\title{
Structural basis for the recognition of the SARS-CoV-2 by full-length human ACE2
}

\author{
Renhong Yan ${ }^{1,2}$, Yuanyuan Zhang, ${ }^{1,2 *}$, Yaning $\mathrm{Li}^{3 *}$, Lu Xia ${ }^{1,2}$, Yingying Guo ${ }^{1,2}$, Qiang Zhou ${ }^{1,2} \dagger$ \\ ${ }^{1}$ Key Laboratory of Structural Biology of Zhejiang Province, Institute of Biology, Westlake Institute for Advanced Study, 18 Shilongshan Road, Hangzhou 310024, Zhejiang \\ Province, China. ${ }^{2}$ School of Life Sciences, Westlake University, 18 Shilongshan Road, Hangzhou 310024, Zhejiang Province, China. ${ }^{3}$ Beijing Advanced Innovation Center for \\ Structural Biology, Tsinghua-Peking Joint Center for Life Sciences, School of Life Sciences, Tsinghua University, Beijing 100084, China. \\ *These authors contributed equally to this work. †Corresponding author. Email: zhouqiang@westlake.edu.cn
}

Angiotensin-converting enzyme 2 (ACE2) is the cellular receptor for SARS coronavirus (SARS-CoV) and the new coronavirus (SARS-CoV-2) that is causing the serious epidemic COVID-19. Here we present cryo-EM structures of full-length human ACE2, in the presence of a neutral amino acid transporter $\mathrm{B}^{\circ} \mathrm{AT} 1$, with or without the receptor binding domain (RBD) of the surface spike glycoprotein (S protein) of SARS-CoV-2, both at an overall resolution of $2.9 \AA$, with a local resolution of $3.5 \AA$ at the ACE2-RBD interface. The ACE2$\mathrm{B}^{\circ} \mathrm{AT} 1$ complex is assembled as a dimer of heterodimers, with the Collectrin-like domain (CLD) of ACE2 mediating homo-dimerization. The RBD is recognized by the extracellular peptidase domain (PD) of ACE2 mainly through polar residues. These findings provide important insights to the molecular basis for coronavirus recognition and infection.

The SARS-CoV-2 is a positive-strand RNA virus that causes severe respiratory syndrome in human. The resulting outbreak of the coronavirus disease 2019 (COVID-19) has emerged as a severe epidemic, claiming more than two thousand lives worldwide between Dec 2019 and Feb 2020 (1, 2). The genome of SARS-CoV-2 shares about $80 \%$ identity with that of SARS-CoV and is about $96 \%$ identical to the bat coronavirus BatCoV RaTG13 (2).

In the case of SARS-CoV, the spike glycoprotein (S protein) on the virion surface mediates receptor recognition and membrane fusion $(3,4)$. During viral infection, the trimeric $S$ protein is cleaved into S1 and S2 subunits and S1 subunits are released in the transition to the post-fusion conformation (47). S1 contains the receptor binding domain (RBD), which directly binds to the peptidase domain (PD) of ACE2 (8), while $\mathrm{S} 2$ is responsible for membrane fusion. When $\mathrm{S} 1$ binds to the host receptor ACE2, another cleavage site on S2 is exposed and is cleaved by host proteases, a process that is critical for viral infection $(5,9,10)$. The $\mathrm{S}$ protein of SARS-CoV-2 may also exploit ACE2 for host infection (2,11-13). A recent publication reported the structure of the S protein of SARS-CoV-2 and showed that the ectodomain of the SARS-CoV-2 S protein binds to the PD of ACE2 with a $K_{d}$ of $\sim 15 \mathrm{nM}(14)$.

Although ACE2 is hijacked by some coronaviruses, its primary physiological role is in the maturation of angiotensin, a peptide hormone that controls vasoconstriction and blood pressure. ACE2 is a type I membrane protein expressed in lungs, heart, kidneys and intestine (15-17). Decreased expression of ACE2 is associated with cardiovascular diseases (1820). Full-length ACE2 consists of an N-terminal PD and a Cterminal Collectrin-like domain (CLD) that ends with a single transmembrane helix and a 40-residue intracellular segment $(15,21)$. The PD of ACE2 cleaves angiotensin (Ang) I to produce Ang-(1-9), which is then processed by other enzymes to become Ang-(1-7). ACE2 can also directly process Ang II to give Ang-(1-7) $(15,22)$.

Structures of the claw-like ACE2-PD alone and in complex with the RBD or the S protein of SARS-CoV have revealed the molecular details of the interaction between the RBD of $\mathrm{S}$ protein and PD of ACE2 (7, 8, 23, 24). Structural information on ACE2 is limited to the PD domain. The single-TM of ACE2 makes it challenging to determine the structure of the fulllength protein.

ACE2 also functions as the chaperone for the membrane trafficking of the amino acid transporter $\mathrm{B}^{0} \mathrm{AT} 1$, also known as SLC6A19 (25), which mediates uptake of neutral amino acids into intestinal cells in a sodium dependent manner. $\mathrm{Mu}$ tations in $\mathrm{B}^{0} \mathrm{AT} 1$ may cause Hartnup disorder, an inherited disease with symptoms such as pellagra, cerebellar ataxia and psychosis (26-28). Structures have been determined for the SLC6 family members dDAT (Drosophila Dopamine transporter) and human SERT (Serotonin transporter, SLC6A4) $(29,30)$. It is unclear how ACE2 interacts with $\mathrm{B}^{0} \mathrm{AT} 1$. The membrane trafficking mechanism for ACE2 and $\mathrm{B}^{\circ} \mathrm{AT} 1$ is similar to the LAT1-4F2hc complex, a large neutral amino acid transporter complex that requires $4 \mathrm{~F} 2 \mathrm{hc}$ for its plasma membrane localization (31). Our structure of LAT1-4F2hc shows that the cargo LAT1 and the chaperone 4F2hc interact through both extracellular and transmembrane domains (32). We reasoned that the structure of the full-length ACE2 may be revealed in the presence of $\mathrm{B}^{0} \mathrm{AT} 1$.

Here we report the cryo-EM structure of the full-length 
human ACE2- $\mathrm{B}^{\circ} \mathrm{AT} 1$ complex at an overall resolution of $2.9 \AA$ and a complex between the RBD of SARS-CoV-2 and ACE2$\mathrm{B}^{0} \mathrm{AT} 1$ complex, also with an overall resolution of $2.9 \AA$ and with $3.5 \AA$ local resolution at the ACE2-RBD interface. The $\mathrm{ACE} 2-\mathrm{B}^{\circ} \mathrm{AT} 1$ exists as a dimer of heterodimers. Structural alignment of the RBD-ACE2- $\mathrm{B}^{0} \mathrm{AT} 1$ ternary complex with the $\mathrm{S}$ protein of the SARS-CoV-2 suggests that two S protein trimers can simultaneously bind to an ACE2 homodimer.

\section{Structural determination of ACE2-B ${ }^{\circ}$ AT1 complex}

The full-length human ACE2 and $\mathrm{B}^{0} \mathrm{AT} 1$, with Strep and FLAG tags on their respective $\mathrm{N}$ terminus, were co-expressed in HEK293F cells and purified through tandem affinity resin and size exclusion chromatography. The complex was eluted in a single mono-disperse peak, indicating high homogeneity (Fig. 1A). Details of cryo-sample preparation, data acquisition, and structural determination are given in the Materials and Methods section of the supplementary material. A 3D reconstruction was obtained at an overall resolution of $2.9 \AA$ from 418,140 selected particles. This immediately revealed the dimer of heterodimers' architecture (Fig. 1B). After applying focused refinement and $\mathrm{C} 2$ symmetry expansion, the resolution of the extracellular domains improved to $2.7 \AA$, while the TM domain remained at $2.9 \AA$ resolution (Fig. 1B, figs. S1 to $\mathrm{S} 3$, and table $\mathrm{S} 1$ ).

The high resolution supported reliable model building. For ACE2, side chains could be assigned to residues 19-768 that contain the PD (residues 19-615) and the CLD (residues 616-768) that consists of a small extracellular domain, a long linker, and the single TM helix (Fig. 1C). Between the PD and the TM is a ferredoxin-like fold domain, we referred to this as the Neck domain (residues 616-726) (Fig. 1C and fig. S4). Homo-dimerization is entirely mediated by ACE2, which is sandwiched by $\mathrm{B}^{0} \mathrm{AT} 1$. Both the PD and Neck domain contribute to dimerization, while each $\mathrm{B}^{0} \mathrm{AT1}$ interacts with the Neck and the TM helix in the adjacent ACE2 (Fig. 1C). The extracellular region is highly glycosylated, with 7 and 5 glycosylation sites on each ACE2 and $\mathrm{B}^{0} \mathrm{AT} 1$ monomer, respectively.

During classification, another subset with 143,857 particles was processed to an overall resolution of $4.5 \AA$. While the Neck domain still dimerizes, the PDs are separated from each other in this reconstruction (Fig. 1D and fig. S1, H to K). We therefore define the two classes as the open and closed conformations. Structural comparison shows that the conformational changes are achieved through rotation of the PD domains, with the rest of the complex nearly unchanged (movie S1).

\section{Homodimer interface of ACE2}

Dimerization of ACE2 is mainly mediated by the Neck domain, with the PD contributing a minor interface (Fig. 2A). We will refer to the two ACE2 protomers as A and B, with residues in protomer $\mathrm{B}$ labeled as residue'. Extensive polar interactions are mapped to the interface between the 2nd (residues 636-658) and the 4th (residues 708-717) helices of the Neck domain (Fig. 2B). Arg652 and Arg710 in ACE2-A form cation- $\pi$ interactions with Tyr641' and Tyr633' in ACE2B. Meanwhile, Arg652 and Arg710 are respectively hydrogenbonded (H-bond) to Asn638' and Glu639', which also interact with Gln653, as does Asn636'. Ser709 and Asp713 from ACE2A are H-bonded to Arg716'. This extensive network of polar interactions indicates stable dimer formation.

The PD dimer interface appears much weaker with only one pair of interactions between Gln139 and Gln175' (Fig. 2C). Gln139 is in a loop which is stabilized by a disulfide bond between Cys133 and Cys141 as well as multiple intra-loop polar interactions (Fig. 2C). The weak interaction is consistent with the ability to transition to the open conformation, in which the interface between the Neck domains remains the same while the PDs are separated from each other by $\sim 25 \AA$ (Fig. 2D and movie S1).

\section{Overall structure of the RBD-ACE2-B ${ }^{\circ}$ AT1 complex}

To gain insight into the interaction between ACE2 and the SARS-CoV-2, we purchased $0.2 \mathrm{mg}$ recombinantly expressed and purified RBD-mFc of the SARS-CoV-2 (for simplicity, we will refer it as RBD) from Sino Biological Inc., mixed it with our purified ACE2-B ${ }^{0} \mathrm{AT} 1$ complex at a stoichiometric ratio of $\sim 1.1$ to 1 , and proceeded with cryo-grid preparation and imaging. Finally, a 3D EM reconstruction of the ternary complex was obtained.

In contrast to the ACE2- ${ }^{\circ} \mathrm{AT} 1$ complex, which has two conformations, "open" and "closed", only the closed state of ACE2 was observed in the dataset for the RBD-ACE2- $\mathrm{B}^{\circ} \mathrm{AT} 1$ ternary complex. The structure of the ternary complex was determined to an overall resolution of $2.9 \AA$ from 527,017 selected particles. However, the resolution for the ACE2-B ${ }^{\circ} \mathrm{AT} 1$ complex was substantially higher than that for the RBDs, which are at the periphery of the complex (Fig. 3A). To improve the local resolution, focused refinement was applied, this allowed us to reach a resolution of $3.5 \AA$ for the RBD, supporting reliable modelling and analysis of the interface (Fig. 3, figs. S5 to S7, and table S1).

\section{Interface between RBD and ACE2}

As expected, each PD accommodates one RBD (Fig. 3B). The overall interface is similar to that between the SARS-CoV and $\operatorname{ACE} 2(7,8)$, mediated mainly through polar interactions (Fig. $4 A$ ). An extended loop region of the RBD spans the archshaped $\alpha 1$ helix of the ACE2-PD like a bridge. The $\alpha 2$ helix and a loop that connects the $\beta 3$ and $\beta 4$ antiparallel strands, referred to as Loop 3-4, of the PD also make limited contributions to the coordination of RBD.

The contact can be divided into three clusters. The two 
ends of the "bridge" interact with the amino $(\mathrm{N})$ and carboxyl (C) termini of the $\alpha 1$ helix as well as small areas on the $\alpha 2$ helix and Loop 3-4. The middle segment of $\alpha 1$ reinforces the interaction by engaging two polar residues (Fig. 4A). At the $\mathrm{N}$ terminus of $\alpha 1$, Gln498, Thr500, and Asn501 of the RBD form a network of hydrogen bonds (H-bonds) with Tyr41, Gln42, Lys353, and Arg357 from ACE2 (Fig. 4B). In the middle of the "bridge", Lys417 and Tyr453 of the RBD interact with Asp30 and His34 of ACE2, respectively (Fig. 4C). At the C terminus of $\alpha 1$, Gln474 of RBD is H-bonded to Gln24 of ACE2, while Phe 486 of RBD interacts with Met82 of ACE2 through van der Waals forces (Fig. 4D).

\section{Comparing the SARS-CoV-2 and SARS-CoV interfaces with ACE2}

Superimposition of the RBD in the complex of SARS-CoV (SARS-CoV-RBD) and ACE2-PD (PDB code: 2AJF) with the RBD in our ternary complex shows that the SARS-CoV-2 RBD (SARS-CoV-2-RBD) is similar to SARS-CoV-RBD with a root mean squared deviation (RMSD) of $0.68 \AA$ over 139 pairs of $\mathrm{C} \alpha$ atoms (Fig. 5A) (8). Despite the overall similarity, a number of sequence variations and conformational deviations are found in their respective interfaces with ACE2 (Fig. 5 and fig. $\mathrm{S} 8$ ). At the $\mathrm{N}$ terminus of $\alpha 1$, the following variations, Arg426 $\rightarrow$ Asn439, Tyr484 $\rightarrow$ Gln498, and Thr487 $\rightarrow$ Asn501, at equivalent positions are observed between SARS-CoV-RBD and SARS-CoV-2-RBD (Fig. 5B). More variations are observed in the middle of the bridge. The most prominent alteration is the substitution of Val404 in the SARS-CoV-RBD with Lys417 in the SARS-CoV-2-RBD. In addition, from SARS-CoV-RBD to SARS-CoV-2-RBD, the substitution of interface residues, Tyr442 $\rightarrow$ Leu455, Leu443 $\rightarrow$ Phe456, Phe $460 \rightarrow$ Tyr473, and Asn $479 \rightarrow$ Gln493, may also change the affinity for ACE2 (Fig. $5 \mathrm{C})$. At the $\mathrm{C}$ terminus of $\alpha 1$, Leu 472 in the SARS-CoV-RBD is replaced by Phe486 in the SARS-CoV-2-RBD (Fig. 5D).

\section{Discussion}

Although ACE2 is a chaperon for $\mathrm{B}^{0} \mathrm{AT} 1$, our focus is on ACE2 in this study. With the stabilization by $\mathrm{B}^{0} \mathrm{AT} 1$, we elucidated the structure of the full-length ACE2. $\mathrm{B}^{0} \mathrm{AT} 1$ is not involved in dimerization suggesting that ACE2 may be a homodimer even in the absence of $\mathrm{B}^{0} \mathrm{AT} 1$. Further examination suggests a dimeric ACE2 can accommodate two $\mathrm{S}$ protein trimers, each through a monomer of ACE2 (fig. S9). The trimeric structure of the S protein of SARS-CoV-2 was recently reported, with one RBD in "up" and two in "down" conformations (PDB code: 6VSB) (14). PD clashes with the rest of the $\mathrm{S}$ protein when the ternary complex is aligned to the RBD of the "down" conformation. There is no clash when the complex is superimposed to the "up" RDB, with RMSD of $0.98 \AA$ over 126 pairs of $\mathrm{C}_{\alpha}$ atoms, confirming that an "up" conformation of $\mathrm{RDB}$ is required to bind to the receptor (fig. S9) (14).
Cleavage of the S protein of SARS-CoV is facilitated by cathepsin L in endosomes, indicating a mechanism of receptormediated endocytosis (10). Further characterization is required to examine the interactions between ACE2 and the viral particle as well as the effect of co-factors on this process $(25,33)$. It remains to be investigated whether there is clustering between the dimeric ACE2 and trimeric S proteins, which may be important for invagination of the membrane and endocytosis of the viral particle, a process similar to other receptor-mediated endocytosis.

Cleavage of the C-terminal segment, especially residues 697 to 716 (fig. S4), of ACE2 by proteases, such as transmembrane protease serine 2 (TMPRSS2), enhances the S proteindriven viral entry $(34,35)$. Residues $697-716$ form the 3 rd and 4th helices in the Neck domain and map to the dimeric interface of ACE2. Thr presence of $\mathrm{B}^{0} \mathrm{AT} 1$ may block the access of TMPRSS2 to the cutting site on ACE2. The expression distribution of ACE2 is broader than $\mathrm{B}^{\circ} \mathrm{AT} 1$. In addition to kidneys and intestine, where $\mathrm{B}^{0} \mathrm{AT} 1$ is mainly expressed, ACE2 is also expressed in lungs and heart (27). It remains to be tested whether $\mathrm{B}^{0} \mathrm{AT} 1$ can suppress SARS-CoV-2 infection by blocking ACE2 cleavage. Enteric infections have been reported for the SARS-CoV, and possibly also for the SARS-CoV-2 $(36,37)$. $\mathrm{B}^{0} \mathrm{AT} 1$ has also been shown to interact with another coronavirus receptor, aminopeptidase N (APN or CD13) (38). These findings suggest that $\mathrm{B}^{0} \mathrm{AT} 1$ may play a regulatory role for the enteric infections of some coronaviruses.

Comparing the interaction interfaces of SARS-CoV-2-RBD and SARS-CoV-RBD with ACE2 reveals some variations that may strengthen the interactions between SARS-CoV-2-RBD and ACE2, and others likely to reduce the affinity compared to SARS-CoV-RBD and ACE2. For instance, the change from Val404 to Lys317 may result in tighter association because of the salt bridge formation between Lys317 and Asp30 of ACE2 (Figs. 4C and 5C). Change of Leu472 to Phe486 may also make stronger van der Waals contact with Met82 (Fig. 5D). However, replacement of Arg426 to Asn439 appears to weaken the interaction by losing one important salt bridge with Asp329 on ACE2 (Fig. 5B).

In summary, our structural work reveals the high-resolution structures of full-length ACE2 in a dimeric assembly. Docking the S protein trimer onto the structure of the ACE2 dimer with RBD of S protein bound suggests simultaneous binding of two $S$ protein trimers to an ACE2 dimer. Structurebased rational design of binders with enhanced affinities to either ACE2 or the S protein of the coronaviruses may facilitate development of decoy ligands or neutralizing antibodies for suppression of viral infection.

\section{REFERENCES AND NOTES}

1. N. Zhu, D. Zhang, W. Wang, X. Li, B. Yang, J. Song, X. Zhao, B. Huang, W. Shi, R. Lu, P. Niu, F. Zhan, X. Ma, D. Wang, W. Xu, G. Wu, G. F. Gao, W. Tan; China Novel Coronavirus Investigating and Research Team, A novel coronavirus from patients with pneumonia in China, 2019. N. Engl. J. Med. 382, 727-733 (2020). 
doi:10.1056/NEJMoa2001017 Medline

2. P. Zhou, X.-L. Yang, X.-G. Wang, B. Hu, L. Zhang, W. Zhang, H.-R. Si, Y. Zhu, B. Li, C.L. Huang, H.-D. Chen, J. Chen, Y. Luo, H. Guo, R.-D. Jiang, M.-Q. Liu, Y. Chen, X.-R. Shen, X. Wang, X.-S. Zheng, K. Zhao, Q.-J. Chen, F. Deng, L.-L. Liu, B. Yan, F.-X. Zhan, Y.-Y. Wang, G.-F. Xiao, Z.-L. Shi, A pneumonia outbreak associated with a new coronavirus of probable bat origin. Nature (2020). doi:10.1038/s41586-0202012-7 Medline

3. T. M. Gallagher, M. J. Buchmeier, Coronavirus spike proteins in viral entry and pathogenesis. Virology 279, 371-374 (2001). doi:10.1006/viro.2000.0757 Medline

4. G. Simmons, P. Zmora, S. Gierer, A. Heurich, S. Pöhlmann, Proteolytic activation of the SARS-coronavirus spike protein: Cutting enzymes at the cutting edge of antiviral research. Antiviral Res. 100, 605-614 (2013). doi:10.1016/i.antiviral.2013.09.028 Medline

5. S. Belouzard, V. C. Chu, G. R. Whittaker, Activation of the SARS coronavirus spike protein via sequential proteolytic cleavage at two distinct sites. Proc. Natl. Acad. Sci. U.S.A. 106, 5871-5876 (2009). doi:10.1073/pnas.0809524106 Medline

6. G. Simmons, J. D. Reeves, A. J. Rennekamp, S. M. Amberg, A. J. Piefer, P. Bates, Characterization of severe acute respiratory syndrome-associated coronavirus (SARS-CoV) spike glycoprotein-mediated viral entry. Proc. Natl. Acad. Sci. U.S.A. 101, 4240-4245 (2004). doi:10.1073/pnas.0306446101 Medline

7. W. Song, M. Gui, X. Wang, Y. Xiang, Cryo-EM structure of the SARS coronavirus spike glycoprotein in complex with its host cell receptor ACE2. PLOS Pathog. 14, e1007236 (2018). doi:10.1371/journal.ppat.1007236 Medline

8. F. Li, W. Li, M. Farzan, S. C. Harrison, Structure of SARS coronavirus spike receptorbinding domain complexed with receptor. Science 309, 1864-1868 (2005). doi:10.1126/science.1116480 Medline

9. J. K. Millet, G. R. Whittaker, Host cell proteases: Critical determinants of coronavirus tropism and pathogenesis. Virus Res. 202, 120-134 (2015). doi:10.1016/i.virusres.2014.11.021 Medline

10. G. Simmons, D. N. Gosalia, A. J. Rennekamp, J. D. Reeves, S. L. Diamond, P. Bates, Inhibitors of cathepsin $L$ prevent severe acute respiratory syndrome coronavirus entry. Proc. Natl. Acad. Sci. U.S.A. 102, 11876-11881 (2005). doi:10.1073/pnas.0505577102 Medline

11. M. Hoffmann, H. Kleine-Weber, N. Krüger, M. Müller, C. Drosten, S. Pöhlmann, The novel coronavirus 2019 (2019-nCoV) uses the SARS-coronavirus receptor ACE2 and the cellular protease TMPRSS2 for entry into target cells. bioRxiv $\begin{array}{lllll}2020.01 .31 .929042 & \text { [Preprint]. } & 31 & \text { January } & 2020 .\end{array}$ https://doi.org/10.1101/2020.01.31.929042.

12. W. Li, M. J. Moore, N. Vasilieva, J. Sui, S. K. Wong, M. A. Berne, M. Somasundaran J. L. Sullivan, K. Luzuriaga, T. C. Greenough, H. Choe, M. Farzan, Angiotensinconverting enzyme 2 is a functional receptor for the SARS coronavirus. Nature 426, 450-454 (2003). doi:10.1038/nature02145 Medline

13. K. Kuba, Y. Imai, S. Rao, H. Gao, F. Guo, B. Guan, Y. Huan, P. Yang, Y. Zhang, W. Deng, L. Bao, B. Zhang, G. Liu, Z. Wang, M. Chappell, Y. Liu, D. Zheng, A. Leibbrandt, T. Wada, A. S. Slutsky, D. Liu, C. Qin, C. Jiang, J. M. Penninger, A crucial role of angiotensin converting enzyme 2 (ACE2) in SARS coronavirusinduced lung injury. Nat. Med. 11, 875-879 (2005). doi:10.1038/nm1267 Medline

14. D. Wrapp, N. Wang, K. S. Corbett, J. A. Goldsmith, C.-L. Hsieh, O. Abiona, B. S. Graham, J. S. McLellan, Cryo-EM structure of the 2019-nCoV spike in the prefusion conformation. Science eabb2507 (2020). doi:10.1126/science.abb2507 Medline

15. M. Donoghue, F. Hsieh, E. Baronas, K. Godbout, M. Gosselin, N. Stagliano, M. Donovan, B. Woolf, K. Robison, R. Jeyaseelan, R. E. Breitbart, S. Acton, A novel angiotensin-converting enzyme-related carboxypeptidase (ACE2) converts angiotensin I to angiotensin 1-9. Circ. Res. 87, E1-E9 (2000). doi:10.1161/01.RES.87.5.e1 Medline

16. H. Zhang, Z. Kang, H. Gong, J. W. Da Xu, Z. Li, X. Cui, J. Xiao, T. Meng, W. Zhou, J. Liu, H. Xu, The digestive system is a potential route of 2019-nCov infection: A bioinformatics analysis based on single-cell transcriptomes. bioRxiv 2020.01.30.927806 [Preprint]. 31 January 2020. https://doi.org/10.1101/2020.01.30.927806.

17. Y. Zhao, Z. Zhao, Y. Wang, Y. Zhou, Y. Ma, W. Zuo, Single-cell RNA expression profiling of ACE2, the putative receptor of Wuhan 2019-nCov. bioRxiv 2020.01.26.919985 [Preprint]. 26 January 2020. https://doi.org/10.1101/2020.01.26.919985.

18. M. A. Crackower, R. Sarao, G. Y. Oudit, C. Yagil, I. Kozieradzki, S. E. Scanga, A. J. Oliveira-dos-Santos, J. da Costa, L. Zhang, Y. Pei, J. Scholey, C. M. Ferrario, A. S. Manoukian, M. C. Chappell, P. H. Backx, Y. Yagil, J. M. Penninger, Angiotensinconverting enzyme 2 is an essential regulator of heart function. Nature 417, 822828 (2002). doi:10.1038/nature00786 Medline

19. L. S. Zisman, R. S. Keller, B. Weaver, Q. Lin, R. Speth, M. R. Bristow, C. C. Canver, Increased angiotensin-(1-7)-forming activity in failing human heart ventricles: Evidence for upregulation of the angiotensin-converting enzyme homologue ACE2. Circulation 108, 1707-1712 (2003). doi:10.1161/01.CIR.0000094734.67990.99 Medline

20. M. K. Raizada, A. J. Ferreira, ACE2: A new target for cardiovascular disease therapeutics. J. Cardiovasc. Pharmacol. 50, 112-119 (2007). doi:10.1097/FJC.0b013e3180986219 Medline

21. H. Zhang, J. Wada, K. Hida, Y. Tsuchiyama, K. Hiragushi, K. Shikata, H. Wang, S. Lin, Y. S. Kanwar, H. Makino, Collectrin, a collecting duct-specific transmembrane glycoprotein, is a novel homolog of ACE2 and is developmentally regulated in embryonic kidneys. J. Biol. Chem. 276, 17132-17139 (2001). doi:10.1074/ibc.M006723200 Medline

22. I. Hamming, M. E. Cooper, B. L. Haagmans, N. M. Hooper, R. Korstanje, A. D. M. E. Osterhaus, W. Timens, A. J. Turner, G. Navis, H. van Goor, The emerging role of ACE2 in physiology and disease. J. Pathol. 212, 1-11 (2007). doi:10.1002/path.2162 Medline

23. R. N. Kirchdoerfer, N. Wang, J. Pallesen, D. Wrapp, H. L. Turner, C. A. Cottrell, K. S. Corbett, B. S. Graham, J. S. McLellan, A. B. Ward, Stabilized coronavirus spikes are resistant to conformational changes induced by receptor recognition or proteolysis. Sci. Rep. 8, 15701 (2018). doi:10.1038/s41598-018-34171-7 Medline

24. P. Towler, B. Staker, S. G. Prasad, S. Menon, J. Tang, T. Parsons, D. Ryan, M. Fisher, D. Williams, N. A. Dales, M. A. Patane, M. W. Pantoliano, ACE2 X-ray structures reveal a large hinge-bending motion important for inhibitor binding and catalysis. J. Biol. Chem. 279, 17996-18007 (2004). doi:10.1074/jbc.M311191200 Medline

25. S. Kowalczuk, A. Bröer, N. Tietze, J. M. Vanslambrouck, J. E. J. Rasko, S. Bröer, A protein complex in the brush-border membrane explains a Hartnup disorder allele. FASEB J. 22, 2880-2887 (2008). doi:10.1096/fi.08-107300 Medline

26. H. F. Seow, S. Bröer, A. Bröer, C. G. Bailey, S. J. Potter, J. A. Cavanaugh, J. E. J. Rasko, Hartnup disorder is caused by mutations in the gene encoding the neutral amino acid transporter SLC6A19. Nat. Genet. 36, 1003-1007 (2004). doi:10.1038/ng1406 Medline

27. R. Kleta, E. Romeo, Z. Ristic, T. Ohura, C. Stuart, M. Arcos-Burgos, M. H. Dave, C. A. Wagner, S. R. M. Camargo, S. Inoue, N. Matsuura, A. Helip-Wooley, D. Bockenhauer, R. Warth, I. Bernardini, G. Visser, T. Eggermann, P. Lee, A. Chairoungdua, P. Jutabha, E. Babu, S. Nilwarangkoon, N. Anzai, Y. Kanai, F. Verrey, W. A. Gahl, A. Koizumi, Mutations in SLC6A19, encoding B0AT1, cause Hartnup disorder. Nat. Genet. 36, 999-1002 (2004). doi:10.1038/ng1405 Medline

28. A. Bröer, K. Klingel, S. Kowalczuk, J. E. J. Rasko, J. Cavanaugh, S. Bröer, Molecular cloning of mouse amino acid transport system $\mathrm{B}^{0}$, a neutral amino acid transporter related to Hartnup disorder. J. Biol. Chem. 279, 24467-24476 (2004). doi:10.1074/ibc.M400904200 Medline

29. A. Penmatsa, K. H. Wang, E. Gouaux, X-ray structure of dopamine transporter elucidates antidepressant mechanism. Nature 503, 85-90 (2013) doi:10.1038/nature12533 Medline

30. J. A. Coleman, E. M. Green, E. Gouaux, X-ray structures and mechanism of the human serotonin transporter. Nature 532, 334-339 (2016) doi:10.1038/nature17629 Medline

31. L. Mastroberardino, B. Spindler, R. Pfeiffer, P. J. Skelly, J. Loffing, C. B. Shoemaker, F. Verrey, Amino-acid transport by heterodimers of 4F2hc/CD98 and members of a permease family. Nature 395, 288-291 (1998). doi:10.1038/26246 Medline

32. R. Yan, X. Zhao, J. Lei, Q. Zhou, Structure of the human LAT1-4F2hc heteromeric amino acid transporter complex. Nature 568, 127-130 (2019). doi:10.1038/s41586-019-1011-z Medline

33. Q. Lin, R. S. Keller, B. Weaver, L. S. Zisman, Interaction of ACE2 and integrin betal in failing human heart. Biochim. Biophys. Acta 1689, 175-178 (2004). doi:10.1016/i.bbadis.2004.05.005 Medline

34. A. Shulla, T. Heald-Sargent, G. Subramanya, J. Zhao, S. Perlman, T. Gallagher, A 
transmembrane serine protease is linked to the severe acute respiratory syndrome coronavirus receptor and activates virus entry. J. Virol. 85, 873-882 (2011). doi:10.1128/JVl.02062-10 Medline

35. A. Heurich, H. Hofmann-Winkler, S. Gierer, T. Liepold, O. Jahn, S. Pöhlmann, TMPRSS2 and ADAM17 cleave ACE2 differentially and only proteolysis by TMPRSS2 augments entry driven by the severe acute respiratory syndrome coronavirus spike protein. J. Virol. 88, 1293-1307 (2014). doi:10.1128/JVI.0220213 Medline

36. C. Drosten, S. Günther, W. Preiser, S. van der Werf, H.-R. Brodt, S. Becker, H. Rabenau, M. Panning, L. Kolesnikova, R. A. M. Fouchier, A. Berger, A.-M. Burguière, J. Cinatl, M. Eickmann, N. Escriou, K. Grywna, S. Kramme, J.-C. Manuguerra, S. Müller, V. Rickerts, M. Stürmer, S. Vieth, H.-D. Klenk, A. D. M. E. Osterhaus, H. Schmitz, H. W. Doerr, Identification of a novel coronavirus in patients with severe acute respiratory syndrome. N. Engl. J. Med. 348, 1967-1976 (2003). doi:10.1056/NEJMoa030747 Medline

37. C. Yeo, S. Kaushal, D. Yeo, Enteric involvement of coronaviruses: Is faecal-oral transmission of SARS-CoV-2 possible? Lancet Gastroenterol. Hepatol. S24681253(20)30048-0 (2020). doi:10.1016/S2468-1253(20)30048-0 Medline

38. J. Jando, S. M. R. Camargo, B. Herzog, F. Verrey, Expression and regulation of the neutral amino acid transporter $\mathrm{B}^{0} \mathrm{AT1}$ in rat small intestine. PLOS ONE 12 , e0184845 (2017). doi:10.1371/journal.pone.0184845 Medline

39. J. Lei, J. Frank, Automated acquisition of cryo-electron micrographs for single particle reconstruction on an FEI Tecnai electron microscope. J. Struct. Biol. 150 , 69-80 (2005). doi:10.1016/i.jsb.2005.01.002 Medline

40. S. Q. Zheng, E. Palovcak, J.-P. Armache, K. A. Verba, Y. Cheng, D. A. Agard, MotionCor2: Anisotropic correction of beam-induced motion for improved cryoelectron microscopy. Nat. Methods 14, 331-332 (2017). doi:10.1038/nmeth.4193 Medline

41. T. Grant, N. Grigorieff, Measuring the optimal exposure for single particle cryo-EM using a $2.6 \AA$ reconstruction of rotavirus VP6. elife 4, e06980 (2015). doi:10.7554/eLife.06980 Medline

42. K. Zhang, Gctf: Real-time CTF determination and correction. J. Struct. Biol. 193, 1-12 (2016). doi:10.1016/i.jsb.2015.11.003 Medline

43. J. Zivanov, T. Nakane, B. O. Forsberg, D. Kimanius, W. J. H. Hagen, E. Lindahl, S. H. W. Scheres, New tools for automated high-resolution cryo-EM structure determination in RELION-3. eLife 7, e42166 (2018). doi:10.7554/eLife.42166 Medline

44. D. Kimanius, B. O. Forsberg, S. H. Scheres, E. Lindahl, Accelerated cryo-EM structure determination with parallelisation using GPUs in RELION-2. eLife 5, e18722 (2016). doi:10.7554/eLife.18722 Medline

45. S. H. Scheres, RELION: Implementation of a Bayesian approach to cryo-EM structure determination. J. Struct. Biol. 180, 519-530 (2012). doi:10.1016/i.jsb.2012.09.006 Medline

46. S. H. Scheres, A Bayesian view on cryo-EM structure determination. J. Mol. Biol. 415, 406-418 (2012). doi:10.1016/i.jmb.2011.11.010 Medline

47. A. Punjani, J. L. Rubinstein, D. J. Fleet, M. A. Brubaker, cryoSPARC: Algorithms for rapid unsupervised cryo-EM structure determination. Nat. Methods 14, 290-296 (2017). doi:10.1038/nmeth.4169 Medline

48. P. B. Rosenthal, R. Henderson, Optimal determination of particle orientation, absolute hand, and contrast loss in single-particle electron cryomicroscopy. J. Mol. Biol. 333, 721-745 (2003). doi:10.1016/i.jmb.2003.07.013 Medline

49. S. Chen, G. McMullan, A. R. Faruqi, G. N. Murshudov, J. M. Short, S. H. W. Scheres, R. Henderson, High-resolution noise substitution to measure overfitting and validate resolution in 3D structure determination by single particle electron cryomicroscopy. Ultramicroscopy 135, 24-35 (2013). doi:10.1016/i.ultramic.2013.06.004 Medline

50. L. G. Trabuco, E. Villa, K. Mitra, J. Frank, K. Schulten, Flexible fitting of atomic structures into electron microscopy maps using molecular dynamics. Structure 16, 673-683 (2008). doi:10.1016/j.str.2008.03.005 Medline

51. P. D. Adams, P. V. Afonine, G. Bunkóczi, V. B. Chen, I. W. Davis, N. Echols, J. J. Headd, L.-W. Hung, G. J. Kapral, R. W. Grosse-Kunstleve, A. J. McCoy, N. W. Moriarty, R. Oeffner, R. J. Read, D. C. Richardson, J. S. Richardson, T. C. Terwilliger, P. H. Zwart, PHENIX: A comprehensive Python-based system for macromolecular structure solution. Acta Crystallogr. D Biol. Crystallogr. 66, 213221 (2010). doi:10.1107/S0907444909052925 Medline
52. P. Emsley, B. Lohkamp, W. G. Scott, K. Cowtan, Features and development of Coot. Acta Crystallogr. D Biol. Crystallogr. 66, 486-501 (2010). doi:10.1107/S0907444910007493 Medline

\section{ACKNOWLEDGMENTS}

We thank the Cryo-EM Facility and Supercomputer Center of Westlake University for providing cryo-EM and computation support, respectively. This work was funded by the National Natural Science Foundation of China (projects 31971123 , 81920108015, 31930059), the Key R\&D Program of Zhejiang Province (2020C04001) and the SARS-CoV-2 emergency project of the Science and Technology Department of Zhejiang Province (2020C03129). Author contributions: Q.Z. and R.Y. conceived the project. Q.Z and R.Y. designed the experiments. All authors did the experiments. Q.Z. R.Y., Y.Z. and Y.L. contributed to data analysis. Q.Z. and R.Y. wrote the manuscript. Competing interests: The authors declare no competing interests. Data and materials availability: Atomic coordinates and cryo EM maps for the ACE2-B AT1 complex of closed conformation (whole structure and map: PDB 6M18 and EMD-30040, extracellular region map: EMD-30044, TM region map: EMD-30045), and the ACE2-B ${ }^{0}$ AT1 complex of open conformation (PDB 6M1D and EMD-30041), and the complex of the RBD of SARS-CoV-2 with the ACE2-B ${ }^{0} \mathrm{AT1}$ complex (whole structure and map: PDB 6M17 and EMD-30039, extracellular region map:EMD30042, TM region map: EMD-30043, ACE2- RBD interface map: EMD-30046) have been deposited in the Protein Data Bank (http://www.rcsb.org) and the Electron Microscopy Data Bank (https://www.ebi.ac.uk/pdbe/emdb/). Correspondence and requests for materials should be addressed to Q.Z. (zhouqiang@westlake.edu.cn).

\section{SUPPLEMENTARY MATERIALS}

science.sciencemag.org/cgi/content/full/science.abb2762/DC1

Materials and Methods

Figs. S1 to S9

Table S1

References (39-52)

MDAR Reproducibility Checklist

Movie S1

12 February 2020; accepted 3 March 2020

Published online 4 March 2020

10.1126/science.abb2762 
A
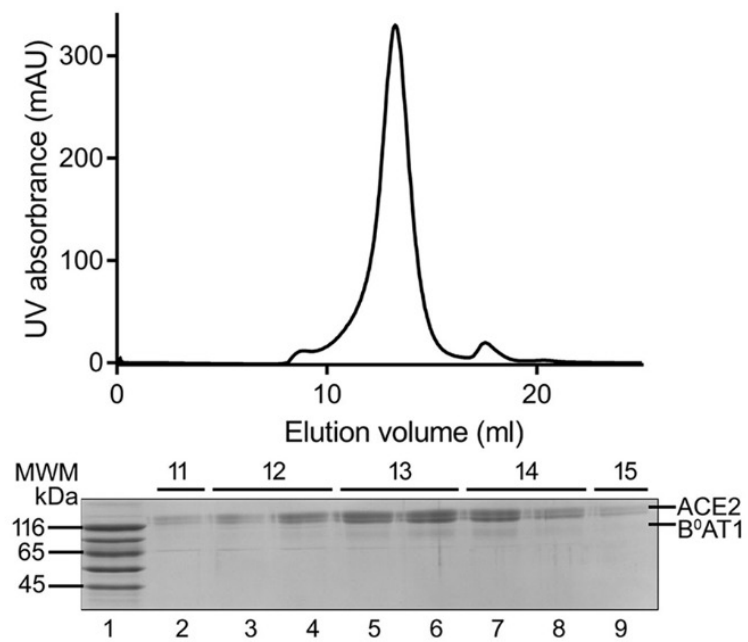

C

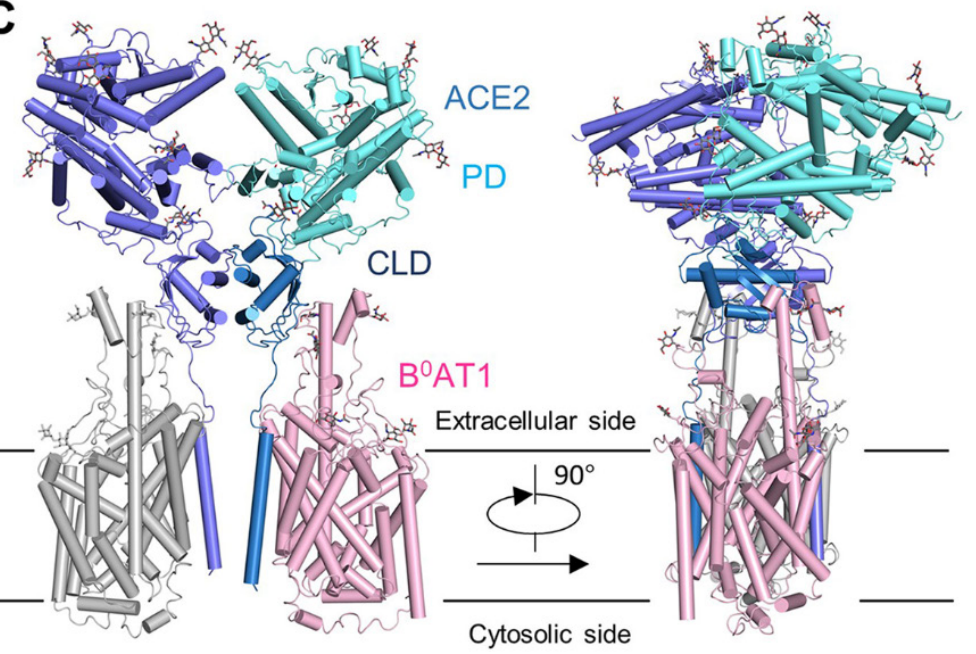

B

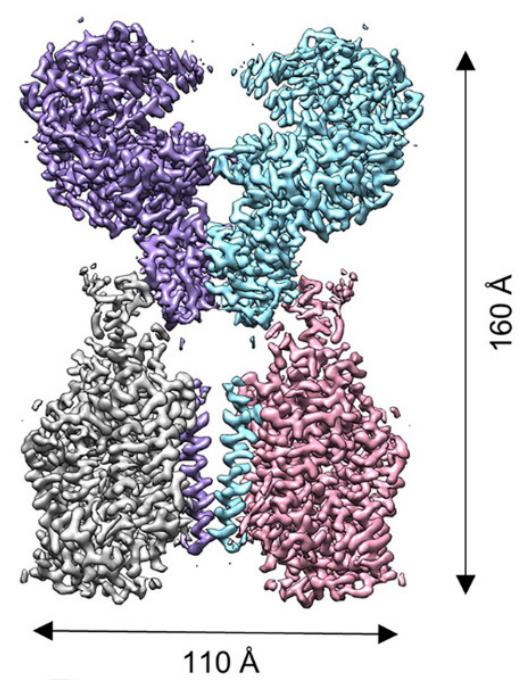

D

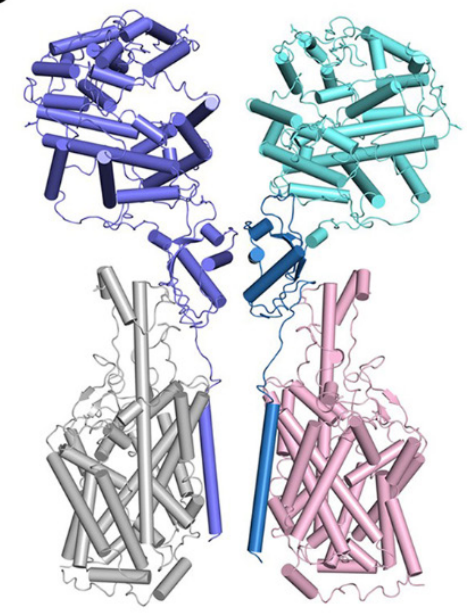

Fig. 1. Overall structure of the $A C E 2-B^{\circ} A T 1$ complex. (A) Representative SEC purification profile of the full-length human ACE2 in complex with $B^{0} A T 1$. (B) Cryo-EM map of the ACE2-B ${ }^{0}$ T1 complex. The map is generated by merging the focused refined maps shown in fig. S2. (C) Cartoon representation of the atomic model of the ACE2-B ${ }^{\circ} A T 1$ complex. The glycosylation moieties are shown as sticks. The complex is colored by subunits, with the protease domain (PD) and the Collectrin-like domain (CLD) in one ACE2 protomer colored cyan and blue, respectively. (D) An open conformation of the ACE2-B ${ }^{\circ} \mathrm{AT} 1$ complex. The two PDs, which contact each other in the "closed" conformation, are separated in the "open" conformation. 
A

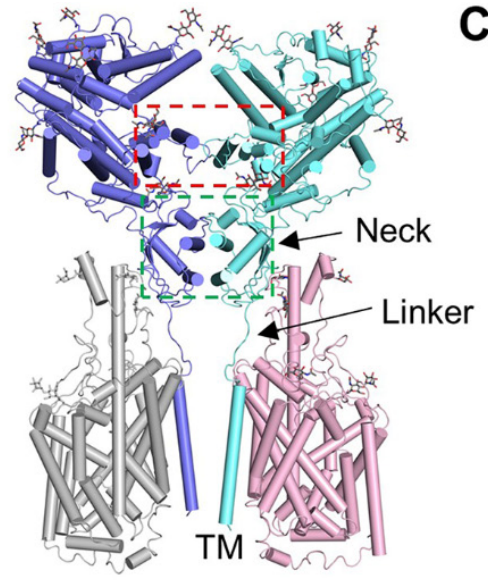

B

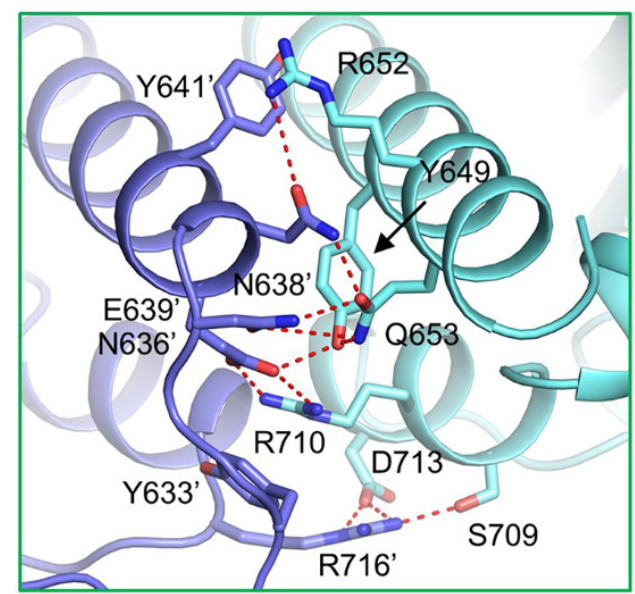

C

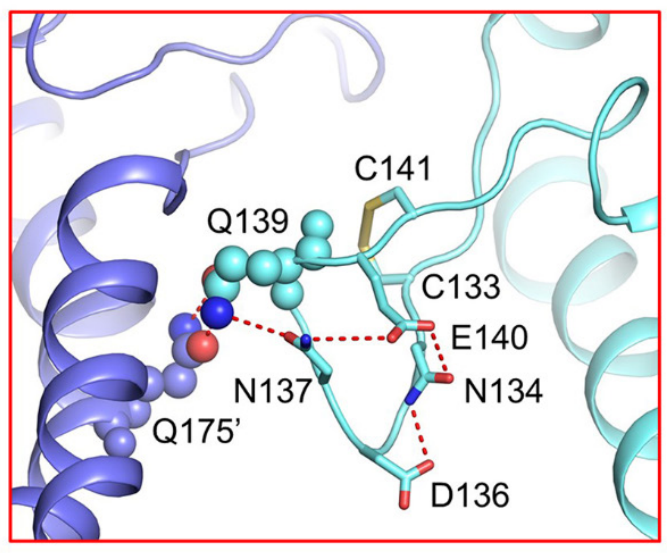

D

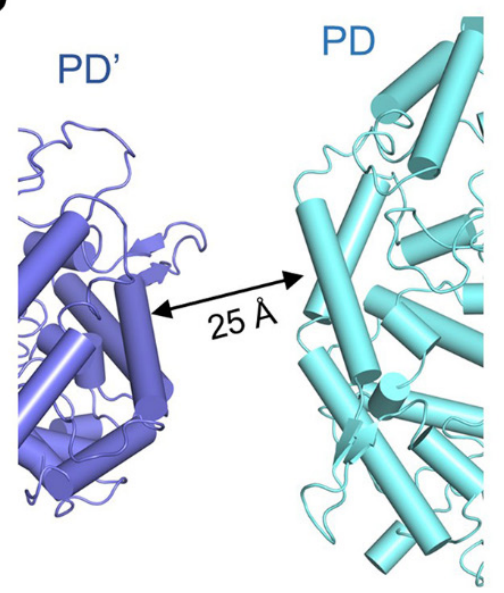

Fig. 2. Dimerization interface of ACE2. (A) ACE2 dimerizes via two interfaces, the $P D$ and the Neck domain. (B) The primary dimeric interface is between the Neck domain in ACE2. Polar interactions are represented by dashed, red lines. (C) A weaker interface between PDs of ACE2. The only interaction is between GIn139 and Gln175', which are highlighted as spheres. The polar residues that may contribute to the stabilization of Gln139 are shown as sticks. (D) The PDs no longer contact each other in the open state.
A

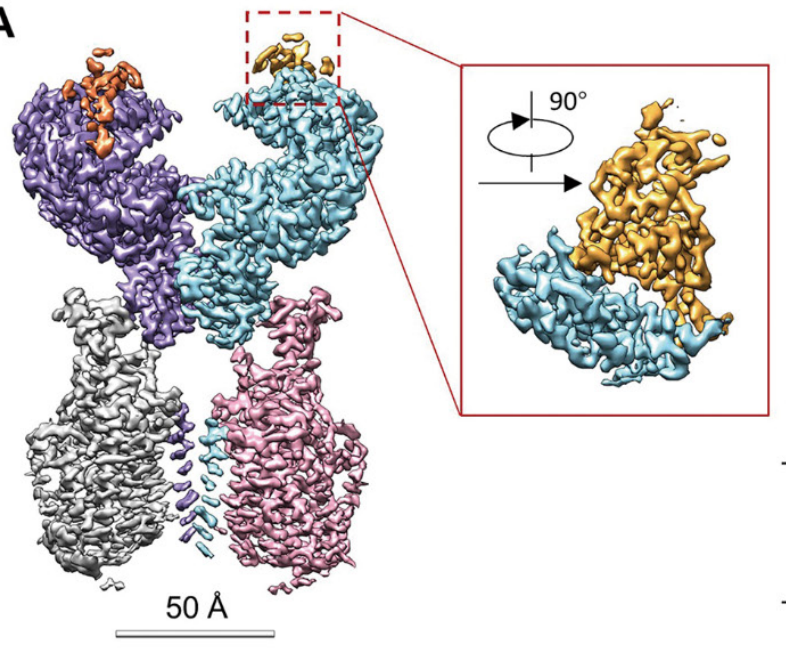

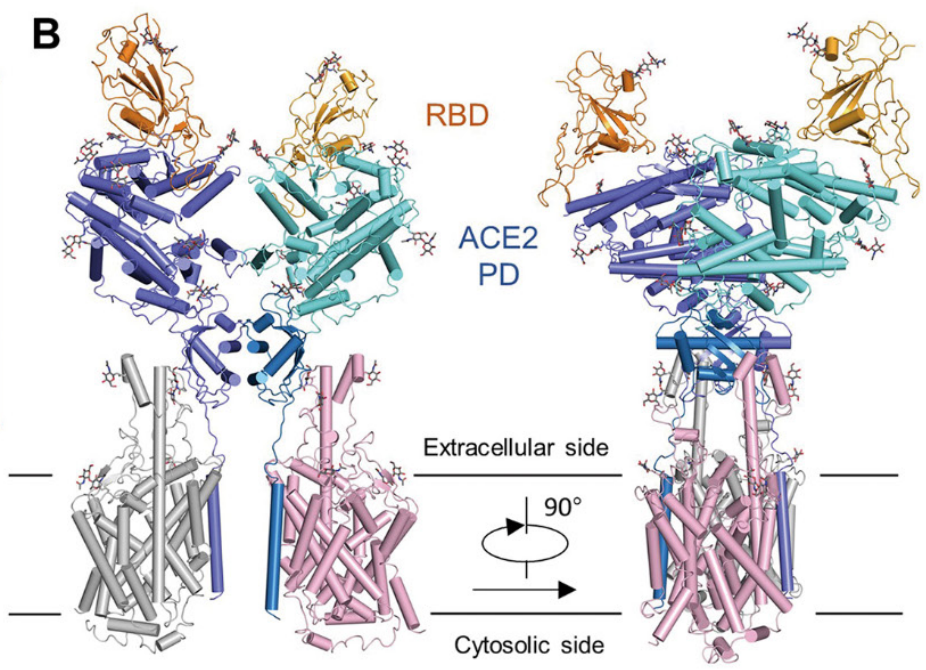

B

RBD 


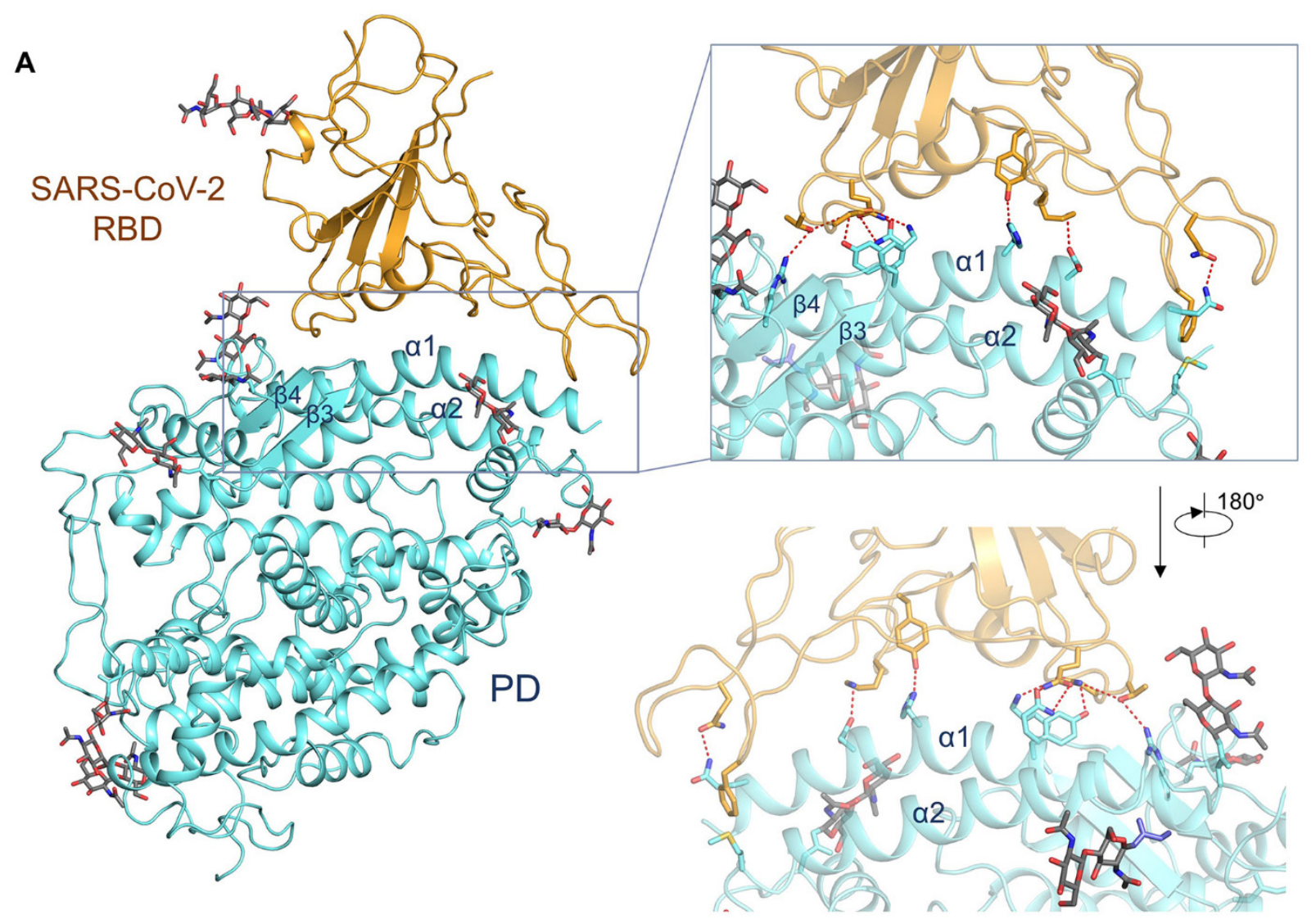

B

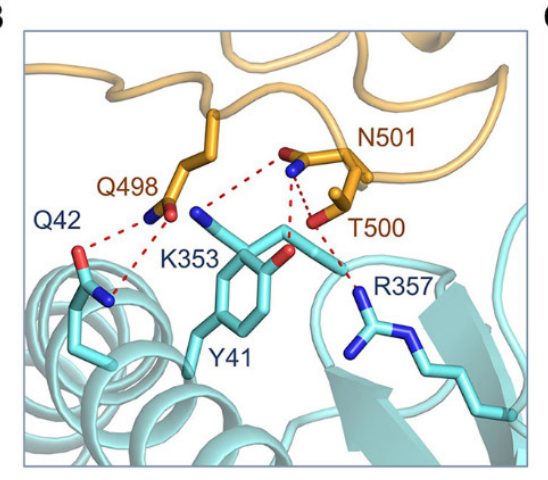

C

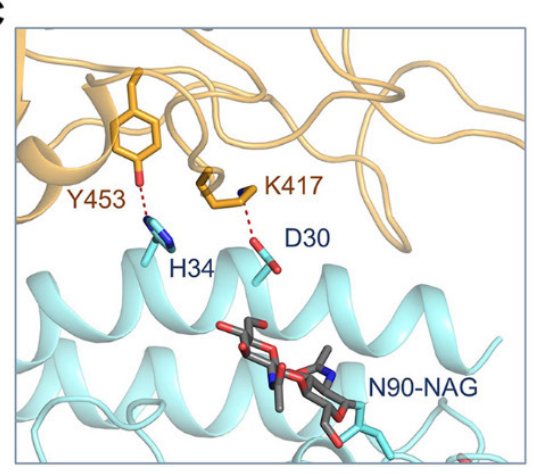

D

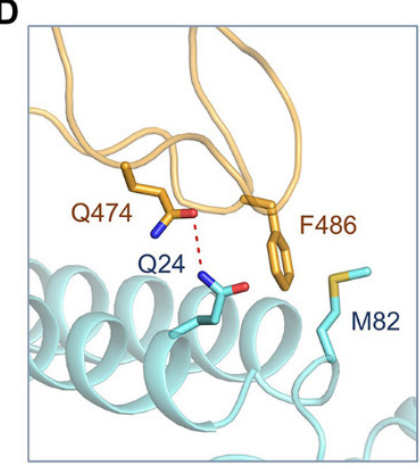

Fig. 4. Interactions between SARS-CoV-2-RBD and ACE2. (A) The protease domain (PD) of ACE2 mainly engages the $\alpha 1$ helix in the recognition of the RBD. The $\alpha 2$ helix and the linker between $\beta 3$ and $\beta 4$ also contribute to the interaction. Only one RBD-ACE2 is shown. (B to D) Detailed analysis of the interface between SARS-CoV-2-RBD and ACE2. Polar interactions are indicated by red, dashed lines. 

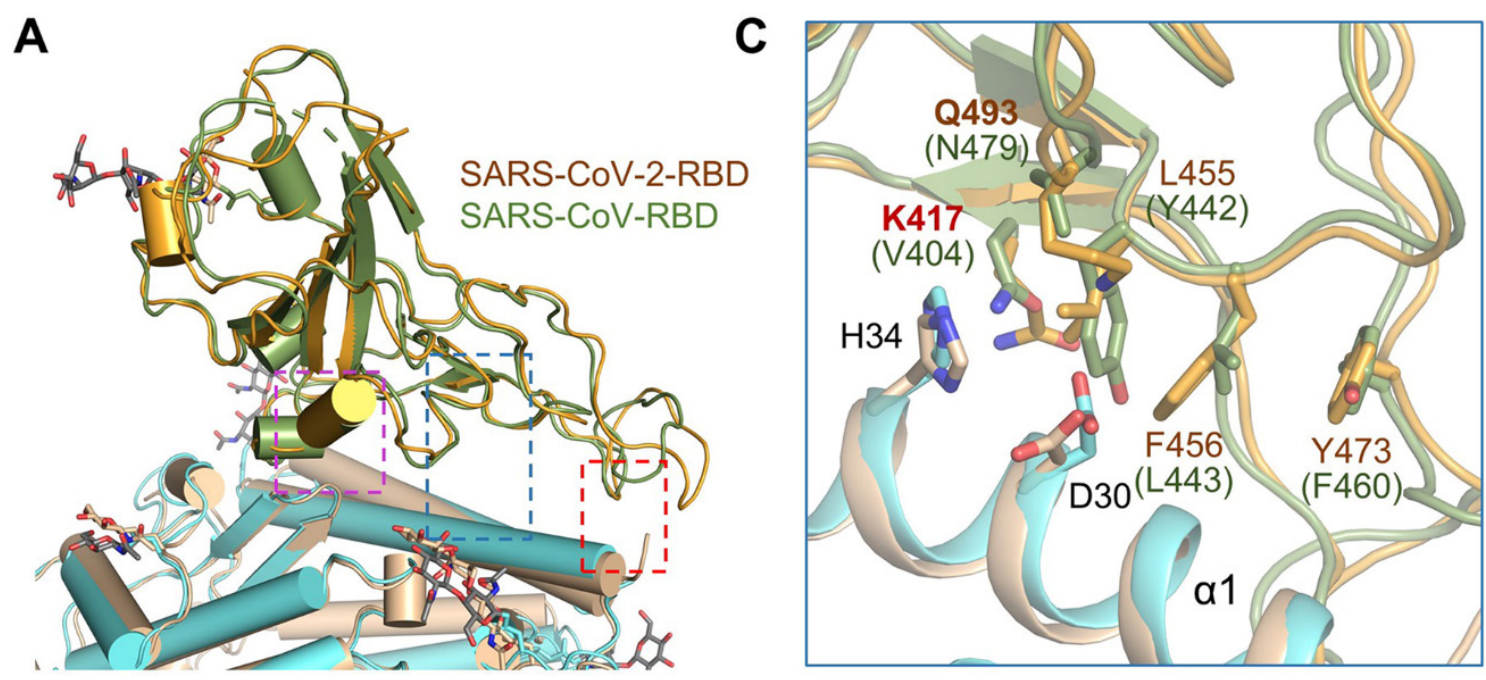

B

D
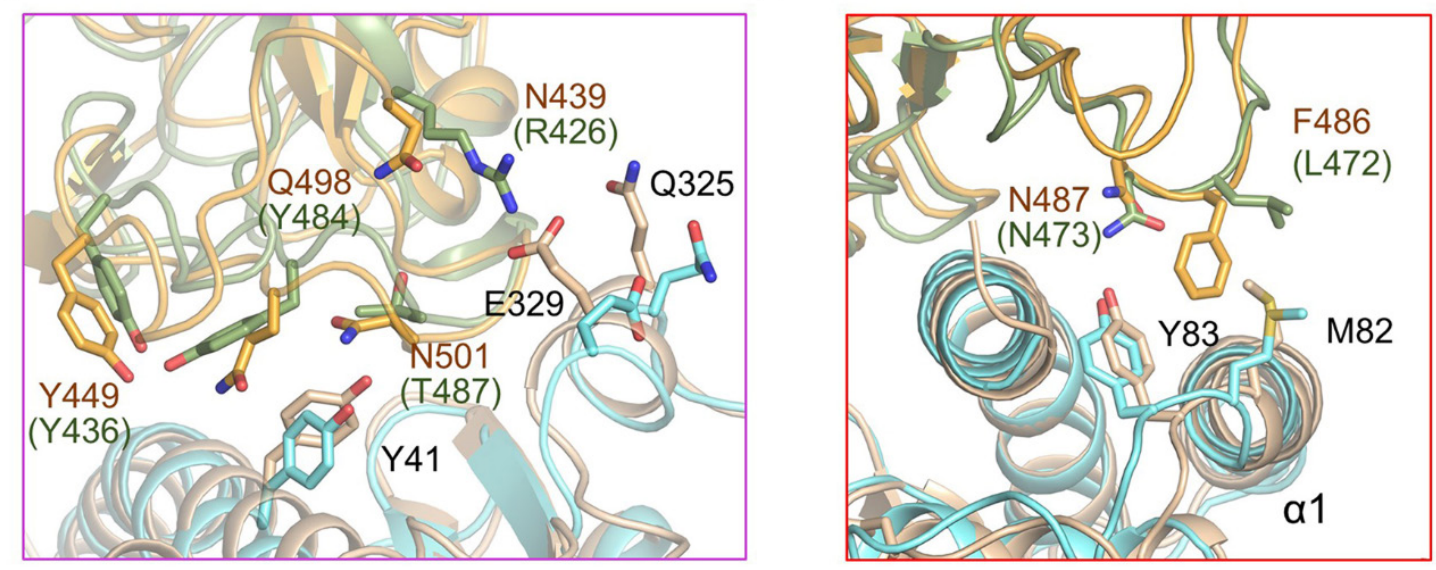

Fig. 5. Interface comparison between SARS-CoV-2-RBD and SARS-CoV-RBD with ACE2. (A) Structural alignment for the SARS-CoV-2-RBD and SARS-CoV-RBD. The complex structure of the ACE2 PD and the SARS-CoV-RBD (PDB code: 2AJF) is superimposed to our cryo-EM structure of the ternary complex relative to the RBDs. The boxed regions are illustrated in detail in (B) to (D). SARS-CoV-2-RBD and the PD in our cryo-EM structure are colored orange and cyan, respectively; SARS-CoV-RBD and its complexed PD are colored green and gold, respectively. (B to D) Variation of the interface residues between SARS-CoV-2-RBD (labeled brown) and SARS-CoV-RBD (labeled green). 